\title{
DE L'IMAGINAIRE AU "TOUT FICTIONNEL"
}

\author{
Marc Augé1
}

Il est évident que le thème du récit médiatique présente un intérêt particulier pour l'ethnologie et pour l'ethnologue, et ce à plusieurs titres, mais principalement parce que l'anthropologie sociale, ou "ethnologie", s'applique plus particulièrement à l'étude des relations d'altérité et à la constitution, dans la pensée et dans la pratique, des relations entre l'un et l'autre, l'un et les autres. Et tout cela est évidemment en jeu dans le récit, et d'une manière particulière dans le récit médiatique.

Je vois en effet plusieurs aspects dans l'intérêt que l'anthropologue peut porter à ce thème. En premier lieu, il est bien évident que tout récit met en jeu une relation sociale minimale. I1 définit des positions : celles de 1'auteur et du narrateur, confondues ou non, celle du récepteur, singulier ou pluriel (et selon les circonstances ce récepteur peut être lecteur, auditeur, spectateur, etc.) ou l'une et l'autre à la fois.

En deuxième lieu se profilent, derrière la question du récit, la question de ce qu'il raconte : témoignage ou imagination, l'un à l'autre mêlés, souvent, et donc, en fin de compte, la question de la fiction.

1 Directeur d'études à l'École des Hautes Études en Sciences Sociales (EHESS) à Paris. 
La fiction d'un point de vue anthropologique est, elle aussi, triplement intéressante :

- par son lien avec l'imagination individuelle qui la conçoit ou qui la reçoit ;

- par son lien avec l'imaginaire collectif qu'elle peut utiliser et relayer, mais qu'elle contribue aussi à enrichir et à modifier ;

- enfin par le rapport qu'elle entretient avec ce qui n'est pas elle mais qui s'y rattache par un aspect ou par un autre : l'histoire, la psychologie, le social, le religieux, etc.

Peut-être peut-on retenir de ce point de vue la formule de Christian Metz, qui écrit dans Le signifiant imaginaire :

La fiction, ce n'est pas d'abord la capacité à inventer des fictions; c'est l'existence historiquement constituée et beaucoup plus généralisée d'un régime de fonctionnement psychique socialement réglé que l'on nomme justement fiction ${ }^{1}$.

C'est dire que le régime de fiction (si je peut utiliser cette expression synthétique) a des conséquences institutionnelles, sociales et pratiques de tous ordres; par exemple, l'existence d'une industrie cinématographique influence les goûts et les imaginations qui, d'un autre côté, l'ont rendu possible.

En troisième lieu, il est bien évident que les médias et les développements technologiques qu'ils connaissent aujourd'hui exercent une influence considérable à la fois sur l'organisation du récit, bien sûr, mais aussi sur la position des acteurs et, aussi, sur le statut de la fiction. Ce qui leur correspond c'est, dans le langage de Metz, un "régime de fiction" spécifique.

Je voudrais essayer d'aborder, de façon relativement précise si possible, la question de savoir si l'excès d'image ne porte pas atteinte à l'imagination et au statut de l'imaginaire au profit d'une invasion de la fiction, d'un passage à ce que j'ai appelé pour faire court le "tout fictionnel" (comme le "tout électrique"...), qui excède d'ailleurs le seul domaine des médias et qui risque d'ôter tout sens, à la limite, à la distinction entre réel et fiction.

Pour ce faire j'examinerai ici trois notions instrumentales/ opératoires que j'essaie de mettre en œuvre dans un travail en cours : le

1 Chr. MET, Le signifiant imaginaire, Paris, Bourgois, 1993 (1977, 1984). 
"triangle de l'imaginaire", la "guerre des rêves" et le "stade de l'écran". Je conclurai en examinant brièvement ce que me paraissent être aujourd'hui les principales modalités de réception et de fabrication de l'image.

\section{Notions opératoires}

\section{Le "triangle de l'imaginaire"}

J'ai essayé par cette notion de représenter le courant (en principe ininterrompu) qui circule, dans les deux sens, entre imagination et fiction, mais aussi entre individu et collectivité. On pourrait ainsi distinguer trois pôles : le pôle de l'imaginaire-mémoire individuel ; celui de l'imaginaire-mémoire-collectif et celui de l'imaginaire-fiction ou fiction, ou encore fiction-création. On dira par exemple que le rêve individuel se situe au pôle imaginaire-mémoire-individuel; que le mythe partagé se situe au pôle imaginaire-mémoire-collectif ; et que le roman se situe au pôle fiction. Mais il devrait rester bien entendu, d'une part que le contenu de chacun de ces pôles ne se limite pas à ces exemples, et d'autre part qu'il est toujours soumis à l'influence des deux autres pôles, qui l'influencent en retour, et qu'en conséquence sont attestées des formes hybrides d'imaginaire qui ne se rattachent exclusivement à aucun des trois pôles.

Pour conforter l'idée d'une circulation intensive entre ces trois pôles, et ceci dans les deux sens, on peut faire appel à quelques témoignages. Ils sont évidemment un peu dispersés... C'est ainsi que, dans un article intitulé Le créateur littéraire et sa fantaisie ${ }^{1}$, Freud tente d'établir une parenté entre la littérature, le jeu de l'enfant et le fantasme de l'adolescent : le créateur littéraire, comme l'adolescent qui fantasme, prend au sérieux sa fantaisie, mais, comme l'enfant qui joue, il n'abandonne pas l'étayage sur le réel; à la différence de l'adolescent qui fantasme, il sépare son monde de fantaisie de la réalité. Freud établit donc un parallèle entre l'enfance, l'adolescence et la création romanesque. Par ailleurs (dans Délire et rêves dans la Gradiva de Jensen ${ }^{2}$ ), il insiste sur le fait que la matière première du psychothérapeute et celle du créateur littéraire sont les mêmes, à cette

1 In L'inquiétante étrangeté et autres essais, trad. française, Paris, Gallimard, 1985.

2 Trad. française, Paris, Gallimard, 1986. 
différence près que le romancier dirige son attention sur son propre inconscient, alors que le psychothérapeute se tourne vers les autres. Si l'on admet donc aisément que l'imaginaire est une source évidente de la fiction, il ne faut pas sous-estimer pour autant le rôle de l'imaginaire et de l'initiative individuelles dans le façonnement de l'imaginaire collectif.

Je pourrais ici citer quelques exemples d'ordre plus ethnographique : Michel Leiris, dans son étude sur la possession et ses aspects théâtraux chez les Éthiopiens de Gondar, insiste sur un aspect très particulier : dans cette région d'Éthiopie, on postule l'existence d'un certain nombre de puissances, d'entités, qui sont en rapport avec les hommes et qu'on appelle les Zars; et ces Zars, moyennant des procédures d'initiation complexes, possèdent un certain nombre d'individus humains. Mais ce phénomène de possession a ceci de paradoxal que chaque Zar ressemble à la personne qu'il possède. Autrement dit, loin d'être une dépossession d'identité, la possession est présentée, en fin de compte, comme renforçant un certain nombre de traits de caractère. L'intéressant, ici, c'est que les péripéties qui peuvent arriver aux individus humains dans telle ou telle occasion entrent dans la mythologie qui parle des Zars en sorte que, finalement, on voit très bien par quel mouvement les péripéties de la vie humaine enrichissent le mythe, qui sera précisément utilisé pour expliquer ce qui peut se passer dans la vie.

C'est un peu dans ce sens, je crois que Georges Devereux, dans son Ethnopsychanalyse complémentariste ${ }^{1}$, disait à propos des Indiens Mohaves que le mythe est efficace parce qu'il a été préalablement rêvé.

Et j'ai remarqué aussi (c'est une indication qui peut-être de quelque intérêt pour d'autres disciplines) que lorsque je travaillais auprès d'un certain nombre de spécialistes, de prêtres du vodu au Togo, de temps en temps des morceaux de mythe étaient utilisés, pour le diagnostic par exemple, parce qu'un mythe n'est jamais donné sous la forme d'un vaste récit avec un début et une fin, comme dans la reconstitution de l'ethnologue : en fait, il est toujours utilisé de manière pragmatique, pour aider à la résolution d'une difficulté particulière. Et le fragment de mythe, cité à l'occasion d'une phrase particulière du rite, faisait évidemment l'objet de commentaires, d'exégèses, de gloses supplémentaires. On voit bien par quel

1 G. DeVereuX, Ethnopsychanalyse complémentariste, Paris, Flammarion, 1972. 
mouvement l'utilisation rituelle qui est faite du mythe l'enrichit au bout du compte de nouveaux développements.

On sait bien également que l'imaginaire collectif informe toujours, de façon plus ou moins marquée, l'imaginaire individuel, d'où la possibilité de faire intervenir la grille symbolique culturelle dans le décryptage des rêves individuels, par exemple. Mais il faut prêter attention également aux liens étroits qui existent entre l'imaginaire collectif et les formes littéraires qui ne relèvent pas, à l'évidence, de la seule initiative individuelle : épopées, légendes, contes dans lesquels Freud voyait des "vestiges déformés de fantaisies, de désirs propres à des nations entières, aux rêves séculaires de la jeune humanité"1.

Au-delà de la beauté de l'expression de Freud sur les rêves séculaires de la jeune humanité, on pourra remarquer l'allusion à la jeunesse ou à l'enfance. Ce qu'il y a de collectif dans les rêves de l'humanité, jeune ou non, c'est peut-être, en effet, une image du commencement et de l'enfance, qui est sans doute d'ailleurs inséparable de celle de la mort. Ce que nous montre l'ethnologie, c'est que, dans beaucoup de sociétés, les enfants sont considérés comme porteurs d'éléments qui proviennent des morts : toute naissance est une réincarnation partielle. Et au niveau individuel, nous savons bien que au souvenir d'enfance est associé le souvenir des morts; des morts alors bien vivants qui entouraient, protégeaient cette enfance. Des historiens du Moyen Age, comme Jacques Le Goff ou JeanClaude Schmitt ${ }^{2}$, l'ont montré à partir de nombreux exemples, comme les récits autobiographiques qui parlent de revenants, ou les récits de rêves ou de visions... Ces historiens nous ont donc montré comment ces récits concouraient à la formation du "moi" et de la subjectivité littéraire.

Carlo Ginzburg s'est interrogé, dans son livre Le sabbat des sorcières $^{3}$ (et il reprenait une interrogation plus ancienne), sur la généralisation à l'Europe entière des thèmes chamaniques sibériens qui impliquent le voyage au pays des morts, la question étant de savoir si cette généralisation, pouvait se faire au nom d'une espèce de diffusion historique ou bien d'un effet de structure, d'une permanence (il a

1 "Le créateur littéraire et la Fantaisie", L'inquiétante étrangeté et autres essais, op. cit. p. 45.

2 J. LE GoFF, L'imaginaire médiéval, Paris, Gallimard, 1985 ; J.-Cl. SCHMITT, Les revenants, Paris, Gallimard, 1994.

3 C. GinzBurg, Le sabbat des sorcières, Paris, Gallimard, 1992. 
évolué sur ce point). I1 se demande, en fin de compte, s'il ne faut pas considérer que l'expérience réelle ou imaginaire de la mort est la matrice de tout récit possible.

Quant au pôle de la fiction, il est bien évident qu'il retentit sur les imaginaires individuel et collectif. Mais on peut se demander, audelà, si l'existence de récits de fiction, par le jeu qu'elle introduit dans le rapport avec le mythe ou avec les croyances, n'instaure pas vis-àvis d'eux une certaine liberté, et pour parler plus précisément une sorte de mixte de distance et de proximité à la fois, une ambivalence donc ; qui serait la condition, par définition problématique, de toute croyance.

C'est un peu ce que suggère Jean-Pierre Vernant à propos des Grecs anciens et classiques, lorsqu'il parle de la croyance et de la fiction comme de deux pôles opposés mais en quelque sorte complémentaires -et notamment dans un article sur les formes de croyance et de rationalité en Grèce. Les premiers poètes étaient des aèdes inspirés qui constituaient en quelque sorte la mémoire du groupe, qui récapitulaient son savoir, et qui en somme en constituaient le ciment social. Puis le statut de la poésie change, mais la confiance à son égard ne diminue pas, alors même que l'on sait bien que ce qu'elle raconte n'est pas absolument réel :

Quel est donc le rapport entre cette confiance qui est faite à ces poètes [cette confiance que je dirais volontiers fondamentale : la confiance en soi-même, en sa propre culture, en sa propre façon de penser] et le fait qu'assez tôt se dégage l'idée que tout cela constitue ce qu'on appelle de la fiction, des choses imaginées, qui ne sont pas le réel ${ }^{1}$.

\section{La "guerre des rêves"}

La deuxième notion, celle de la "guerre des rêves", pourrait nous servir à situer le problème de l'image dans le contexte d'affrontement et de domination qui est souvent le sien. Nous savons que les sociétés vivent aussi par l'imaginaire, un imaginaire qui ne s'apparente pas seulement à la fiction gratuite, mais aussi à la nécessité de symboliser le monde, l'espace, le temps pour les comprendre ; d'où, dans tous les

\footnotetext{
1 J.-P. VERNANT, "Formes de croyance et de rationalité en Grèce", Archives de sciences sociales des religions, $\mathrm{n}^{\circ} 163,1987, \mathrm{p} .115-123$.
} 
groupes sociaux, l'existence de mythes, de récits, d'inscriptions symboliques de divers ordres, de repères qui organisent les rapports entre les uns et les autres. Dans les situations de contact culturel (expression un peu pudique pour désigner souvent des phénomènes de colonisation et d'affrontement), l'un des terrains de rencontre et d'affrontement est donc naturellement et précisément celui de l'imaginaire. Les stratégies de conversion s'apparentent parfois à une guerre des images, qu'il est nécessaire de gagner pour réduire chez les autres le noyau de l'ultime résistance, pour le convaincre totalement. Les historiens du Moyen Age que je citais à l'instant ont étudié ces stratégies, les stratégies de l'Église pour affermir en Europe le socle du christianisme. Mais je pense plus particulièrement à un historien comme Serge Gruzinski (dans un livre qu'il a d'ailleurs intitulé $L a$ guerre des images ${ }^{1}$ ) qui nous montre de la façon la plus éclairante, sur l'exemple du Mexique du XVI $\mathrm{XI}^{\mathrm{e}}$ siècle et des siècles suivants, comment opère une telle stratégie, qui est littéralement une stratégie de substitution : les ordres mendiants, puis les jésuites ont tenté, non sans succès, de substituer des images chrétiennes aux images, très sophistiquées elles-mêmes, d'une tradition amérindienne -qui était sur ce point très élaborée. Dans tous les cas la stratégie vise, par différents moyens, à repousser l'imaginaire collectif qui est en place lorsqu'arrivent les conquistadors vers la position de la fiction, où il peut se reconvertir sous forme de légendes, de folklore, de curiosité, de couleur locale, en lui substituant les nouvelles images liées aux nouvelles croyances. Je passe sur cette longue histoire ; elle entraîne, comme on le sait et comme on peut le concevoir aisément, des résistances, des réinterprétations. D'où souvent un phénomène qu'on pourrait dire "de surimpression", le basculement n'étant jamais total. L'Église n'est jamais sûre de la conversion des Amérindiens (ou de sa nature), elle ne l'est toujours pas et les Indiens à la longue impriment leur propre imagination au catholicisme venu d'ailleurs.

La figure du "désenchantement du monde", bien appréciée des sociologues, obéit au même dispositif : elle tente d'évacuer la religion chrétienne et les restes du paganisme vers le pôle de la fiction et d'imposer ses propres mythes, sa vision de l'avenir, aux mythes du passé. Tel est au moins le modèle. On sait que dans la réalité, il n'y a pas non plus, tant s'en faut, de substitution pure et simple, mais des phénomènes de surimpression, là encore liés à la résistance des uns,

1 S. GRUZINSKI, La guerre des images, Paris, Fayard, 1990. 
aux échecs des autres et à la nécessaire coexistence, en fin de compte, entre ces trois pôles de l'imaginaire.

Au bout du parcours, on pourrait se demander si la situation d'actuelle n'a pas quelque chose d'inédit et d'indépassable.

Quelque chose d'inédit parce qu'on voit s'amorcer un nouveau phénomène de substitution inachevée, en ce sens que les mythes de la modernité, les mythes d'avenir liés à l'idée de progrès, ce que Lyotard appelait les "grands récits", sont bien évacués du côté de la fiction (on nous dit qu'ils sont morts), mais rien ne les remplace. En outre la situation d'après-modernité est caractérisée par une surabondance d'images qui ne prétendent pas, à première vue, dessiner les linéaments d'un nouvel imaginaire collectif.

Quelque chose d'indépassable, peut-être, à cause de la coexistence immobiliste de ce vide de l'imaginaire collectif et du trop plein d'images. Pour le dire autrement, nous pouvons avoir le sentiment d'être colonisés, mais sans savoir par qui, colonisés par l'image mais sans savoir d'où elle vient ni même ce qu'elle représente. Pour préciser ce point, il faut me semble-t-il évoquer la troisième notion instrumentale : le "stade de 1'écran".

\section{Le "stade de l'écran"}

Que faut-il entendre par "stade de l'écran"? Si je me réfère à Christian Metz, que j'ai cité plus tôt, sûrement pas l'équivalent du "stade du miroir", car ce n'est pas une image de mon propre corps que je vois sur l'écran du cinéma ou de la télévision. Quant au phénomène d'identification dont le cinéma est le lieu, il se construit moins autour d'un sujet-objet (ce "moi" qui "est un autre") qu'autour d'un sujet pur, d'un sujet, nous dit Christian Metz, "(...) qui se situe au point de fuite de la perspective monoculaire reprise à la peinture par le cinéma".

C'est donc le dispositif cinématographique lui-même, ce regard sans visage, auquel s'identifie le spectateur. Metz, à la faveur d'une comparaison entre état filmique et état onirique, propose en outre deux remarques qui peuvent retenir notre attention. La première nous invite à discerner dans l'attitude du spectateur de cinéma un "retrait narcissique", dit-il, et un abaissement des défenses du "moi". La seconde, à l'inverse, porte sur un effet qui se distingue à la fois de l'identification au film comme dispositif, comme instance voyante, et 
de l'identification secondaire au personnage : le sujet percevant reconnaît alors l'existence d'un Autre, l'auteur, analogue à lui-même, analogue au "je", sujet de la perception, et l'effet propre du "stade du miroir" s'inverse.

Dans le "stade de l'écran" (par nature exceptionnel parce que des images en sont l'instrument et que le fantasme des autres ne nous est ordinairement pas sympathique, ne nous retient pas) c'est l'autre qui devient un "je".

Lorsque la convenance des images filmiques au fantasme du spectateur peut être constatée par lui, la satisfaction, le sentiment d'un petit miracle (comme dans l'état de passion amoureuse lorsqu'il est partagé) tient à une sorte d'effet, rare par nature, qui peut se définir comme la rupture d'une très ordinaire solitude.

La fiction, on le voit dans cette version optimiste mais exceptionnelle du "stade de l'écran", peut donc être pour l'imagination et la mémoire d'un individu l'occasion d'éprouver l'existence d'autres imaginaires que le sien -mais analogues au sien. Et cette expérience repose à la fois sur l'existence d'une fiction reconnue comme telle ; d'une vue sur le réel qui ne se confond pas avec lui, ni avec les imaginaires collectifs qu'il interprète; et sur l'existence d'un auteur reconnu comme tel, avec son caractère singulier, et instituant de ce fait avec chacun de ceux qui constituent son public (et aussi entre eux) un lien virtuel de socialisation.

C'est aujourd'hui sur ces deux points (le statut de la fiction et la place de l'auteur) que nous pouvons nous interroger. Et je voudrais le faire ici à partir de deux points de vue complémentaires : par rapport à la réception des images et par rapport à leur conception.

\section{Réception des images}

Si nous reprenons les analyses de Metz, force nous est de reconnaître qu'il n'y a pas de différence entre l'état filmique et l'état télévisuel. Au premier abord on pourrait même penser que les défenses du "moi" sont moins menacées par le second que par le premier. Le dispositif télévisuel est en effet différent du dispositif filmique :

- le regard du spectateur ne s'y identifie pas à l'instance voyante, comme dans une salle de projection; le décor quotidien reste en 
place, appartement ou bistrot, et la lumière allumée, le plus souvent ;

- l'échange des propos avec l'entourage est toujours possible, l'écran est petit, à hauteur de regard, du moins pour l'instant (les écrans grandissent...) ; l'identification aux personnages, lorsque des films ou des téléfilms passent sur le petit écran (bien nommé !) est pour cet ensemble de raisons moins immédiate : la possibilité de changer de canal, de zapper, confère en première apparence au téléspectateur un pouvoir de choix et de décision qui n'a pas d'équivalent du côté du spectateur de film au cinéma, même si celui-ci peut toujours sortir de la salle...

Mais tout ce qui distingue la télévision du cinéma ouvre en même temps d'autres possibilités d'hallucination, plus insidieuses.

Tout d'abord la télévision est quotidienne et familière ; pour beaucoup elle est le principe organisateur du temps (chaque jour, chaque semaine, chaque année) : comme les premiers clochers catholiques, elle ponctue les heures du jour. Comme toute liturgie, elle annonce les offices de la semaine. Comme toute religion, elle se modèle sur le rythme saisonnier de l'année. Elle introduit de ce fait, dans le foyer, des visages d'autant plus familiers qu'on les attend à heures fixes et qu'on peut avoir le sentiment de les avoir choisis, au besoin en changeant de chaîne au bon moment. La maison se peuple ainsi de dieux lares, de divinités domestiques aimables, d'humeur toujours égale, rassurantes. Les silhouettes masculines ou féminines qui animent les jeux, présentent la météorologie ou commentent l'actualité deviennent à la longue aussi indispensable au foyer que ceux qui y vivent réellement. Elles cristallisent des sympathies, parfois des antipathies; elles sont rarement indifférentes. Elles sont des stars sans être des comédiens, comme si le petit écran assurait d'emblée à ceux qui s'y manifestent régulièrement un statut de personnage fictionnel analogue à celui des acteurs qui l'ont conquis de haute lutte, sous les lumières des sunlights et grâce à une saine gestion de leur vie privée et de sa médiatisation.

En deuxième lieu, c'est le statut même de la fiction qui est moins bien assuré à la télévision qu'au cinéma. L'écart entre fiction et réel s'y fait moins perceptible ; c'est déjà le cas avec ces stars du quotidien que je viens d'évoquer. Certaines d'entre elles nous présentent les aspects les plus quotidiens de la réalité qui nous entoure : le temps qu'il fait, les faits divers, l'actualité politique, les résultats sportifs. Mais c'est à titre de stars, de personnages déjà fictionnels, qu'elles 
pénètrent notre intimité quotidienne. Le choix d'une chaîne pour le journal télévisé est souvent commandé par l'image du présentateur. Ce léger flou dans la distinction réel-fiction s'étend à d'autres aspects de la perception télévisuelle. Je vais en citer en vrac quelques manifestations...

Les héros de séries télévisées américaines ont une telle présence à l'écran que le personnage, dans leur cas, absorbe tout ce qui touche à l'acteur. Bien sûr, certains acteurs arrivent à changer de rôle, et parfois même à passer du petit au grand écran (on en a des exemples), mais l'indissociabilité du personnage et de l'acteur reste l'élément clé des séries télévisées : cette indissociabilité qui leur confère d'ailleurs une dimension mythique, une silhouette, un caractère, peu de psychologie, a pour corollaire l'extrême réalisme du décor et des situations : les commissariats de police, les cabinets d'avocats, les tribunaux des séries américaines sont des copies conformes de la réalité. Les fictions qu'elles mettent en scène ont des allures de reportage.

I1 faudrait citer en troisième lieu tous les cas de fictionnalisation (de mise en fiction donc) du réel, dont la télévision est un instrument essentiel et qui correspondent à une véritable révolution dès lors que ce n'est plus la fiction qui imite le réel mais, semble-t-il, le réel qui reproduit la fiction. Cette mise en fiction est d'abord liée à l'abondance d'images et à l'abstraction du regard qui en procède. L'image télévisée égalise les événements sans pouvoir jouer, comme la presse écrite, de la mise en page, de la différence des caractères, etc.

De temps à autre, toute référence à une quelconque réalité disparaît. Ainsi la publicité joue-t-elle des effets supposés de son insistance antérieure et procède-t-elle par allusions, par citations auto référencées : c'est simplement le début d'un refrain, l'esquisse d'une image qui sont censés nous rappeler des séquences complètes et audelà (ou en deçà) l'excellence d'un café ou d'une automobile.

L'effet d'égalisation ne joue pas seulement sur les situations, mais aussi sur les personnes et les personnages : dans l'Olympe télévisée, on retrouve côte à côte, mais pêle-mêle, et parfois véritablement au contact, les vedettes de la politique, du spectacle, du sport, mais aussi bien les marionnettes qui les imitent, les personnages qui leur ressemblent et les journalistes qui les présentent. Tous stars et personnages fictionnels au sens de Christian Metz et, qui plus est, obligés d'exister comme personnages fictionnels pour exister comme personnalités politiques, artistiques, voir scientifiques. L'important n'est pas ce que pensent ces personnalités de leur passage par la fiction, mais 
l'effet produit par cette égalisation fictionnelle sur ceux qui accèdent au monde extérieur principalement par la T.V. Les effets d'égalisation peuvent être imputés pour une bonne part à l'image elle-même, dès lors que les médias sont condamnés à les multiplier, à produire et reproduire de l'image sans interruption (sans transition, comme disent les présentateurs). Mais l'image n'est plus seule en cause lorsque l'actualité entend l'utiliser et en quelque sorte se définir à travers elle. Lors de la guerre du Golfe et plus tard lors du débarquement américain en Somalie, nous étions presque convoqués devant nos postes de télévision pour assister à l'heure dite, en direct, au début des opérations.

J'en suis resté jusqu'ici à l'image perçue, aux modalités de réception de l'image. D'une certaine manière nous ne sommes pas encore très loin de la position du spectateur de cinéma. Mais pourtant nous constatons, et c'est déjà beaucoup, que la frontière entre réel et fiction se fait moins nette et que l'auteur, même s'il existe, même quand il existe, est absent de la conscience du téléspectateur. Mais d'autres signes indiquent que la fictionnalisation du monde est en route et qu'elle ne passe pas seulement par l'image télévisée...

\section{Production des images}

Il faut constater que, non contents de percevoir les images, les humains en général, (les mâles en particulier, parce que l'appareil de prise de vues leur devient aussi indispensable que le volant de l'automobile), les amateurs, les touristes se mettent à en produire, non point tant pour faire une œuvre, pour faire de l'art (encore qu'on se soit déjà interrogé sur ce thème) que pour accumuler les témoignages de leur passage dans les lieux qu'ils ont à peine eu le temps de voir.

Comme si ce parcours au futur antérieur ne prenait sens qu'au retour, dans les soirées passées à regarder, en compagnie de parents ou d'amis résignés à jouer ce rôle de témoin, le film d'une pérégrination passée. S'achève alors le mouvement au terme duquel la vérité de ce que le sujet a vécu ou n'a pas vécu, et du sujet lui-même (car certains artifices ou la complaisance d'un témoin lui permettent de figurer sur la diapositive ou dans le film) se trouve transporté de l'image et de l'écran qui lui sert de support. Il ne s'agit donc plus maintenant, si je reprends à ce propos l'expression "stade de l'écran", d'évoquer l'effet de brusque sympathie qui fait d'un auteur 
l'équivalent d'un "je", mais d'un face-à-face, décalé dans le temps, par lequel le sujet s'identifie à une image passée de lui-même. " «Je» est un autre", peut-être, mais cet autre n'est plus là. Le monde de son côté est chaque jour d'avantage aménagé pour être visité, mais plus encore filmé et en fin de compte projeté sur écran : chaque nuit, les hauts lieux du monde s'illuminent, mais de plus en plus on nous y propose le spectacle de ce que nous venons nous-mêmes y chercher, des images ; il faudrait parler du Mont-Saint-Michel, de Waterloo, etc.

L'entreprise de fictionnalisation, qu'encouragent ces premiers succès, se fait du coup plus ambitieuse : elle crée en rase campagne des univers nouveaux, des parcs d'amusement, des fictions sans récits, simplement encombrées de quelques récits résidus et de quelques appels publicitaires. Disneyland en est l'archétype : une fausse rue de village américain, un faux saloon, un faux Mississippi, des personnages de Disney qui courent dans tous ces faux lieux, un faux château et sa belle au bois dormant composent le décor d'une fiction au troisième degré. La fiction, notamment celle des contes européens, avait été portée à l'écran par Disney, et la voilà qui revient sur terre pour se faire visiter! Images d'images d'images... Alors que vont faire les visiteurs? Mais ils vont filmer, bien sûr ! Filmer pour remettre dans leur boîte noire tous les personnages qui n'auraient pas dû en sortir, mais en profitant de l'occasion pour les rejoindre ou pour leur adjoindre à tout le moins la présence de leurs proches : femme, enfants, qui tous ensemble pourront se voir bientôt à l'écran, sur l'écran de la T.V. avec Mickey, Donald et le Prince Charmant. Les parcs d'amusement, les clubs de vacances, les parcs de loisirs et de résidence comme les Center Parcs, mais aussi les villes privées qui voient le jour, de plus en plus nombreuses, en Amérique, et même ces résidences fortifiées et protégées qui s'élèvent dans toutes les villes du tiers-monde comme autant de châteaux forts, constituent ce qu'on pourrait appeler des "bulles d'immanence". On trouve encore d'autres bulles d'immanence, par exemple les grandes chaînes hôtelières ou commerciales qui reproduisent peu ou prou le même décor, distillent le même type de musique à travers leurs rayons ou dans leurs ascenseurs, diffusent les mêmes films vidéos et proposent les mêmes produits aisément identifiables d'un bout de la terre à l'autre. Au fond, les bulles d'immanence sont l'équivalent fictionnel des cosmologies : 
- elles sont constituées d'une série de repères plastiques, architecturaux, musicaux, textuels, qui permettent de s'y reconnaître ;

- elles dessinent et marquent une frontière au-delà de laquelle elles ne répondent plus de rien ;

- elles sont à la fois plus matérielles et plus lisibles que les cosmologies (qui sont des visions symbolisées du monde), leur apprentissage est plus aisé mais il leur manque évidemment une symbolique, un mode prescrit de relation aux autres (réduit dans leur cas à un code de bonne conduite pour les usagers) et un système d'interprétation de l'événement (même si elles s'emploient à constituer des mondes en réduction : microcosmes du macrocosme où est proclamée la dignité du consommateur qui les fréquente);

- elles restent des parenthèses, que l'on peut ouvrir ou fermer à discrétion, moyennant finances et la connaissance de quelques codes élémentaires.

La fiction dès lors devient encore plus hardie : non contente de créer des parenthèses nouvelles, elle s'attaque au réel lui-même pour le subvertir et le transformer.

Elle entreprend de remodeler, selon ses critères, les formes de la ville. La presse a signalé il y a quelques mois, que Disney Corporation (comme promoteur) avait été vainqueur d'un concours organisé par la mairie et l'État de New York pour l'édification d'un hôtel et d'un centre de commerce et de loisirs à Time Square et pour la restauration de l'hôtel presque centenaire New Amsterdam, dans la 42ème rue de Manhattan. Disney Corporation semblait également devoir être chargée de développer un programme de loisirs à Central Park et de créer un grand magasin où l'on trouverait tous les sousproduits de ses films au 711 de la 5ème Avenue. C'est donc les deux architectes de Disney qui ont gagné ce concours. Et le plus remarquable dans ce projet, c'est qu'il installe en pleine ville, comme une composante normale de celle-ci, le monde de Superman (hôtels garnis d'écrans), monde de Superman qui lui-même avait été conçu comme une imitation de la ville, une fiction dans la ville fictive! Les deux architectes victorieux ont opté pour une esthétique du chaos, mais c'est très délibérément un chaos de $\mathrm{BD}$ et de dessin animé. D'ailleurs, certains journalistes l'ont noté, ce projet en cours à Time Square est fidèle à l'esthétique des centres de loisirs déjà installés aux ÉtatsUnis ; une esthétique qui se tient éloignée des débats sophistiqués sur le sens de l'œuvre, comme si déjà l' "effet Disney" se prenait au 
sérieux et se prenait pour référence, se constituait en auto référence pour l'avenir. On y est donc : c'est la fiction qui imite la fiction.

La question peut en définitive se poser de savoir si toutes les relations qui s'établissent à travers les médias, quelles que soient leur éventuelle originalité, ne relèvent pas d'abord d'un déficit symbolique, d'une difficulté à créer du lien social in situ. Le "moi" fictionnel, comble d'une fascination qui s'amorce dans toute relation exclusive à l'image, est un "moi" sans relation, et du même coup sans support identitaire (toute identité se construisant évidemment à travers des altérités), susceptible dès lors d'absorption par le monde d'images où il croit pouvoir se trouver et se reconnaître.

Je ne voudrais pas conclure sur cette note désespérée. Si la fictionnalisation du présent se substitue ou s'ajoute aujourd'hui à la mythification de l'histoire, au premier enchantement du monde (qui passe par la mythification des origines) et au second (la mythification de l'avenir), s'il est dans sa logique de produire un "moi" également fictionnel et incapable d'inscrire sa réalité et son identité dans une relation effective aux autres, il nous faut définir non seulement (comme le faisait le héros de Calderone dans Le monde est un rêve) une morale d'attente en cas de réveil, mais peut-être une morale de résistance, et s'appuyer sur quelques constats simples.

En premier lieu, il ne faut pas confondre les modèles et la réalité : de même que nous pouvons ramasser à la pelle des exemples d'enchantement du monde, y compris dans les secteurs les plus technologisés des sociétés les plus avancées, comme on dit, de même nous devons savoir que les notions de désenchantement, de fin des grands récits, de post- ou de sur-modernité et de fictionnalisation renvoient à des modèles qui sont des vues partielles sur un réel, qui les autorise mais ne se confond avec aucun d'entre eux.

En second lieu, l'image est une image: quelle que soit sa puissance, elle n'a que les vertus qu'on lui prête. Elle peut séduire sans aliéner, pour autant que tout un système ne s'emploie pas à en faire un instrument de décérébration. Le sort de l'image ne lui appartient pas.

Qui seront demain les résistants?

Je pense d'abord à tous ceux qui ne renoncent ni à l'histoire passée ni à l'histoire à venir, et qui dénoncent l'“idéologie du présent", dont l'image peut-être un relais puissant. Tous les créateurs qui, maintenaient vaille que vaille la circulation entre les trois pôles que je signalais (imaginaire-mémoire-individuel, imaginaire- 
mémoire-collectif et fiction, qui au terme du mouvement actuel se réduirait à un seul : la fiction, image devant laquelle se trouverait seul le "moi" un peu perdu), tous les créateurs donc qui ne renonceront pas à susciter le miracle de la rencontre.

Et puis tous les rêveurs (la nuit est importante !) assez habiles à cultiver leurs propres fantasmes pour tourner en dérision intime le prêt-à-porter imaginaire des illusionnistes du "tout fictionnel".

En somme tous ceux qui se soucient d'avantage de construire la modernité que de la court-circuiter.

Il faut donc veiller, dans tous les sens du terme, c'est-à-dire aussi rêver. 